\title{
FXR and JAK step up to BAT
}

Brown adipose tissue (BAT) produces heat through uncoupled electron transport in the mitochondria. Two new papers describe potential therapeutic avenues to increase the amount of energy burned by BAT, one through administration of a farnesoid $\mathrm{X}$ receptor (FXR) agonist in the intestine and the other through inhibition of Janus kinase/signal transducer and activator of transcription (JAK/STAT) signalling directly in adipocytes. These discoveries could lead to new therapeutics to treat obesity.

Systemic FXR agonists are currently being investigated as potential treatments for hypercholesterolaemia and other diseases. However, they increase weight gain and worsen glucose intolerance in mice with dietinduced obesity (DIO). Fang et al. therefore investigated the effects of oral treatment with fexaramine, an FXR agonist that is absorbed poorly by the intestine. Fexaramine had substantially different effects to systemic FXR agonists. In DIO mice, gutrestricted treatment with fexaramine reduced weight gain and systemic inflammation, and increased the thermogenic activity of BAT (as measured by oxygen consumption and body temperature).
The authors attribute these effects primarily to secretion of the endocrine hormone fibroblast growth factor 15 (FGF15; the human homologue is FGF19) by intestinal cells after FXR engagement. FGF15 is known to induce BAT thermogenesis and alter bile acid production in hepatocytes. Fexaramine-treated mice also had a shift in the serum bile acid composition, which could be caused to some extent by FGF15.

Although the systemic effects of fexaramine treatment could be partially attributable to changes in serum bile acids, they could also be caused by changes in BAT. Indeed, adipocytes from fexaramine-treated DIO mice were phenotypically similar to brown adipocytes and expressed BAT-associated genes. Although the mechanism through which fexaramine induced these changes is not completely known, increased $\beta$-adrenergic signalling, possibly downstream of FGF15, is likely to have a role. In a second paper, Moisan et al. developed a screen to identify compounds that promote white-to-brown conversion in human adipocytes. White adipocytes were treated with small molecules that promote this conversion and assayed for the expression of uncoupling protein 1 (UCP1), a mitochondrial protein characteristic of BAT. Hits from an initial screen of 867 small molecules were then assayed for their capacity to change the distribution of lipid droplets within the adipocytes; brown adipocytes are characterized by lipid droplets that are more numerous and smaller than those in their white counterparts. In these screens, inhibitors of Janus kinase 3 (JAK3) increased the expression of UCP1 and promoted BAT morphology. The effects of JAK3 inhibitors were subsequently validated in other cell culture systems, including mouse white adipose tissue explants.

JAK3 inhibitors increased the cellular mitochondrial content and rate of respiration, but cells maintained a white-like gene expression profile. Notably, the brown-like characteristics were maintained after removal of drug, suggesting that JAK3 inhibition induces a stable change in metabolism without altering cell fate.

The acquisition of brown-like characteristics by white adipocytes, perhaps by JAK3 inhibition or oral fexaramine treatment, is a promising new avenue in the treatment of obesity and related metabolic diseases.

Megan Cully

ORIGINAL RESEARCH PAPERS Fang, S. et al. Intestinal FXR agonism promotes adipose tissue browning and reduces obesity and insulin resistance. Nature Med. http://dx.doi.org/10.1038/ nm.3760 (2015) | Moisan, A. et al. White-to-brown metabolic conversion of human adipocytes by JAK activation. Nature Cell Biol. 17, 56-67 (2014) 